\title{
NON-LOXODROMIC SUBSTITUTIONS AND GROUPS IN $N$ DIMENSIONS *
}

BY

EDWARD B. VAN VLECK

Linear substitutions

$$
x^{\prime}=\frac{a x+b}{c x+d}
$$

are commonly divided into two classes, loxodromic and non-loxodromic, and the non-loxodromic substitutions are further classified as elliptic, parabolic, and hyperbolic. So far as I am aware, no corresponding classification of projective substitutions in $n-1$ nonhomogeneous or $n$ homogeneous variables has been suggested. $\dagger$ The classification which is introduced below is based upon the formulation of a projective substitution under the shape of a homogeneous substitution with determinant +1 . If the substitution (1) is written in homogeneous form

$$
x_{1}^{\prime}=a x_{1}+b x_{2}, \quad x_{2}^{\prime}=c x_{1}+d x_{2}
$$

with unit determinant, the substitution is non-loxodromic when, and only when, $a+d$ is real; that is, when the characteristic equation

$$
\left|\begin{array}{cc}
a-\lambda & b \\
c & d-\lambda
\end{array}\right|=0
$$

is a real equation. The substitution is then hyperbolic, parabolic, or elliptic $\ddagger$ according as the two roots of this equation are real and distinct, coincident, or imaginary. Correspondingly, the projective substitutions are taken $e x-$ clusively in this paper in homogeneous form,

$$
\begin{aligned}
& x_{1}^{\prime}=a_{11} x_{1}+a_{12} x_{2}+\cdots+a_{1 n} x_{n}, \\
& \cdot \cdot \cdot \cdot \cdot \cdot \cdot \cdot \cdot \cdot \cdot \\
& x_{n}^{\prime}=a_{n 1} x_{1}+a_{n 2} x_{2}+\cdots+a_{n n} x_{n},
\end{aligned}
$$

* Presented to the Society, March 25, 1921.

† Fubini in his Introduzione alla Teoria dei Gruppi discontinui e delle Funzioni automorfe, pp. 77-8, has applied the above terms to those substitutions which are movements of

$$
x_{n}^{2}+\epsilon\left(x_{1}^{2}+x_{2}^{2}+\cdots+x_{n-1}^{2}\right)=1 \quad(\epsilon= \pm 1),
$$

into itself. His aims and distinctions are very different from those of this paper.

$\ddagger$ An application of the terms elliptic and hyperbolic differing slightly from the above usage of Klein is also to be found in the literature. Cf. Veblen's Projective Geometry, vol. 2, pp. 5, $171,248$. 
with determinant $\Delta$ equal to +1 . I call a substitution non-loxodromic, if, when so written, it possesses a real characteristic equation,

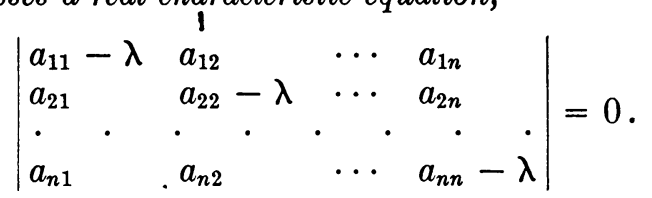

The characteristic roots are accordingly real or pairs of conjugate imaginaries.

It is well known that there are just two classes of groups (1) in a single variable which consist of non-loxodromic substitutions without a common pole. Either all the substitutions of the group leave invariant a circle in the complex $x$-plane and the group is called a "group with a principal circle" (Hauptkreis Gruppe), or the group consists entirely of elliptic substitutions. In the former case the group can be so transformed that the circle becomes the real axis. We have then a canonical form for the group in which its substitutions have real coefficients and unit-determinant, and, conversely, any group of this character is non-loxodromic. A second canonical form is

$$
x^{\prime}=\frac{(a+b i) x+(c+d i)}{(c-d i) x+(a-b i)} \quad\left(a^{2}+b^{2}-c^{2}-d^{2}=1\right),
$$

which leaves the unit circle invariant. The second class of non-loxodromic groups-the elliptic groups-can be put into a canonical form in which the substitutions are all of form

$$
x^{\prime}=\frac{(a+b i) x+(c+d i)}{-(c-d i) x+(a-b i)} \quad\left(a^{2}+b^{2}+c^{2}+d^{2}=1\right),
$$

and the group can be interpreted as a group of rotations of a sphere into itself.

When the number of homogeneous variables is greater than 2 , there are, of course, more varieties of non-loxodromic substitutions to be distinguished than the familiar hyperbolic, elliptic and parabolic types, and it was perhaps scarcely to be expected that the result summarized in the preceding paragraph would have its exact analogue. Nevertheless an exactly similar result is obtained. There are still just two types of non-loxodromic groups. A limitation upon this statement (perhaps unnecessary) is introduced by the proof which requires that the non-loxodromic group should contain at least one substitution having a characteristic equation with $n$ distinct roots. Corresponding to the hypothesis of the preceding paragraph that the substitutions (1) should not have a common pole, we have now the requirement that our non-loxodromic group of substitutions (2) in $n$ homogeneous variables should not leave invariant a linear space of less than $n-1$ (complex) dimensions; in other words, should not transform into itself a hyperplane nor a space common to two or more hyperplanes in our projective space $S_{n-1}$. 
One normal form obtained for the first class of non-loxodromic groups is that in which all substitutions (2) of the group have real coefficients and a determinant equal to +1 . In this normal form the group obviously leaves invariant the real space embedded in the complex space $S_{n-1}$ of the $n$ homogeneous complex coördinates which is of $2 n-2$ real dimensions. Any projective $(n-1)$-dimensional image of this real space within the complex space has been called a "chain" (von Staudt, Pieri), and every non-loxodromic group of our first class accordingly leaves some chain invariant. If $n-1$ is even, all projective substitutions which leave a chain invariant form a nonloxodromic group of the first class. When $n-1$ is odd, this is not the case. The most inclusive non-loxodromic group which leaves the chain invariant is then a subgroup of the set of all substitutions (2) which leave the chain invariant.*

The second class of non-loxodromic groups is novel and interesting. It occurs only when $n$ is an even number $2 m$. Any group of this class can be so transformed that all its substitutions have simultaneously the canonical form

$$
\begin{aligned}
& x_{2 i-1}^{\prime}=\left(a_{2 i-1,1} x_{1}+a_{2 i-1,2} x_{2}\right) \\
& +\cdots+\left(a_{2 i-1,2 m-1} x_{2 m-1}+a_{2 i-1,2 m} x_{2 m}\right), \\
& x_{2 i}^{\prime}=\left(-\bar{a}_{2 i-1,2} x_{1}+\bar{a}_{2 i-1,1} x_{2}\right) \\
& +\cdots+\left(-\bar{a}_{2 i-1,2 m} x_{2 m-1}+\bar{a}_{2 i-1,2 m-1} x_{2 m}\right) \\
& \quad(i=1, \cdots, m),
\end{aligned}
$$

where $\bar{a}_{i j}$ denotes the conjugate of $a_{i j}$. Thus the determinant of the substitution consists of $m^{2}$ blocks of form

$$
\left|\begin{array}{cc}
a_{i j} & a_{i, j+1} \\
-\bar{a}_{i, j+1} & \bar{a}_{i j}
\end{array}\right| \quad \text { (odd } i \text { and } j \text { ), }
$$

similar to the block of coefficients of (4). It is shown that when a determinant has this structure, it is necessarily positive or zero. $\dagger$

For any (non-singular) substitution of the second class the elementary divisors of (3) occur only in conjugate pairs $\left(\lambda-\lambda_{i}\right)^{s},\left(\lambda-\bar{\lambda}_{i}\right)^{s}$ of equal degree, this statement applying as well for real roots $\lambda_{i}$ which furnish pairs of equal elementary divisors. Conversely, a non-singular substitution which fulfills this requirement is one of the second class and may be transformed into the normal form (5). Such substitutions will be called elliptic (restrictedly elliptic, when the characteristic roots are $m$ distinct pairs of conjugate imag-

* J. W. Young in these T r a n s a c t i o n s, vol. 11 (1910), p. 280, investigates the necessary and sufficient condition that a collineation $(n=3)$ shall leave a two-dimensional chain invariant.

† This result has been known for the special case in which all the elements of the determinant are real. Cf. Kowalewski, Einführung in die Determinantentheorie, pp. 156-158. 
inaries). The characteristic property of an elliptic substitution in its normal form (5) is that it converts any pair of points

$$
P=\left(x_{1}, x_{2} ; \cdots ; x_{2 m-1}, x_{2 m}\right), \quad Q=\left(-\bar{x}_{2}, \bar{x}_{1} ; \cdots ;-\bar{x}_{2 m}, \bar{x}_{2 m-1}\right)
$$

into another pair of points whose coördinates are similarly related, any substitution having this property being necessarily of form (5). The invariant points of any substitution (5) occur in such pairs; in particular, the $2 m$ invariant points of a restrictedly elliptic substitution, taken in normal form, consist of $m$ distinct pairs.

It will be noticed that in (5) the $2 m$ equations of the substitution are associated in pairs. In its normal form the most general group of the second or elliptic type comprises all non-singular elliptic substitutions (5) with the same pairing of the coördinates.

\section{DeRIVATION OF TWO STANDARD FORMS FOR NON-LOXODROMIC GROUPS}

As remarked in the introduction, we will suppose our projective substitutions to be written throughout in homogeneous form (2) with determinant

$$
\Delta \equiv\left|\begin{array}{llll}
a_{11} & a_{12} & \cdots & a_{1 n} \\
\cdot & \cdot & \cdot & \cdot \\
a_{n 1} & a_{n 2} & \cdots & a_{n n}
\end{array}\right|
$$

equal to +1 . Obviously the necessary and sufficient condition that a substitution (2) should be non-loxodromic by the above definition is that the sum of all the principal minors of each order $r \leqq n$ should be real.

When the roots of the characteristic equation (3) are all distinct, the substitution can be transformed through an appropriate substitution $T$ into the canonical form

$$
x_{i}^{\prime}=\lambda_{i} x_{i} \quad(i=1, \cdots, n) \quad\left(S_{1}\right) .
$$

We will treat only groups of non-loxodromic substitutions which contain at least one such substitution $S_{1}$, and we will suppose that the group has been so transformed that $S_{1}$ has the form (8). Further, it will be assumed that the subscripts $i$ have been so assigned in (8) that for $i=1,2, \cdots, 2 m \leqq n$ the $\lambda_{i}$ are successively $m$ pairs of conjugate imaginaries, while the remaining $\lambda_{i}$ are real.

Consider now any non-loxodromic substitution $S$, given by (2), which combines with $S_{1}$ so as to generate a non-loxodromic group. The sum of principal minors $a_{11}+a_{22}+\cdots+a_{n n}$ is real, likewise the corresponding sum

$$
\lambda_{1}^{k} a_{11}+\lambda_{2}^{k} a_{22}+\cdots+\lambda_{n}^{k} a_{n n} \equiv r_{k}
$$

for $S S_{1}^{k}$. Denoting these real sums by $r_{k}$ and taking $k=0,1, \cdots, n-1$, we have a system of $n$ equations linear in the $a_{i i}$. The determinant of the 
system is the product $\Pi\left(\lambda_{i}-\lambda_{j}\right)$ which cannot vanish in consequence of our hypothesis of the distinctness of the roots of $S_{1}$. There is therefore a unique solution for the $a_{i i}$ in terms of the $\lambda_{i}, r_{i}$. The first $2 m$ coefficients $\lambda_{i}^{k}$ in each equation are successively pairs of conjugate imaginaries while the remaining ones are real. If, therefore, we exchange each of the first $m$ odd terms on the left side of each equation with the following even term, we have a system of equations which is the conjugate of the preceding system, $a_{i i}$ and $a_{i+1, i+1}$ being interchanged for odd $i<2 m$. Consequently $a_{i i}$ and $a_{i+1, i+1}$ are conjugate imaginaries for odd values of $i<2 m$, while the $a_{i i}$ are real for $i>2 m$.

Consider next the combination of (2) with any substitution

$$
x_{i}^{\prime}=b_{i 1} x_{1}+b_{i 2} x_{2}+\cdots+b_{i n} x_{n}
$$

of the same non-loxodromic group. The product $S^{\prime} S$ is a substitution

$$
x_{i}^{\prime}=B_{i 1} x_{1}+B_{i 2} x_{2}+\cdots+B_{i n} x_{n} \quad(i=1, \cdots, n),
$$

in which

$$
B_{i j}=b_{i 1} a_{1 j}+b_{i 2} a_{2 j}+\cdots+b_{i n} a_{n j} .
$$

Suppose first $m \geqq 1$. Then $B_{11}, B_{22}$ are conjugate imaginaries, and the same is true of the corresponding coefficients

$$
\begin{aligned}
& B_{11}^{(k)}=\lambda_{1}^{k} b_{11} a_{11}+\lambda_{2}^{k} b_{12} a_{21}+\cdots+\lambda_{n}^{k} b_{1 n} a_{n 1}, \\
& B_{22}^{(k)}=\lambda_{1}^{k} b_{21} a_{12}+\lambda_{2}^{k} b_{22} a_{22}+\cdots+\lambda_{n}^{k} b_{2 n} a_{n 2}
\end{aligned}
$$

in $S^{\prime} S_{1}^{k} S$. Take $k=0,1, \cdots, n-1$. We have then in (11) two systems of equations linear in the $b_{1 i} a_{i 1}$ and $b_{2 i} a_{i 2}$ respectively with a common determinant $\Pi\left(\lambda_{i}-\lambda_{j}\right)$ not zero. In the two systems corresponding coefficients $\lambda_{i}^{k}$ are the same, the first $m$ pairs being conjugate imaginaries and the remaining ones real, while the corresponding left members are conjugate imaginaries. We may therefore regard the two systems of equations as conjugate imaginary systems in which the $b_{1 i} a_{i 1}$ and $b_{2 i} a_{i 2}$ correspond to one another for $i>2 m$, while for odd $i<2 m$ they correspond respectively to $b_{2, i+1} a_{i+1,2}$ and $b_{1, i+1} a_{i+1,1}$ respectively. The solution of the two systems therefore gives

$$
\begin{array}{ll}
b_{1 i} a_{i 1}=\bar{b}_{2, i+1} \bar{a}_{i+1,2} & (\text { odd } i<2 m), \\
b_{2 i} a_{i 2}=\bar{b}_{1, i+1} \bar{a}_{i+1,1} & (\text { odd } i<2 m), \\
b_{1 i} a_{i 1}=\bar{b}_{2 i} \bar{a}_{i 2} & (i>2 m) .
\end{array}
$$

We will now make the Fundamental Hypothesis that for each value of $i \leqq n$ there is in the non-loxodromic group at least one substitution (2) for which $a_{1 i}$ is not zero and also one for which $a_{i 1}$ is not, leaving for later con- 
sideration the geometrical significance of this hypothesis. Choose then two substitutions $S, S^{\prime}$ for which $b_{21} a_{12} \neq 0$. Then by (13) $b_{12}$ and $a_{21}$ are not zero. Taking next $S^{\prime}$ identical with $S$, we see by (13) that $a_{12} a_{21}$ is its own conjugate and hence is real. A very different outcome is obtained according as we suppose that this product is positive or negative. The two corresponding cases we will refer to as case 1 and case 2 respectively.

To normalize the groups in these two cases it will suffice to make a simple change of variable

$$
x_{i}=\rho_{i} y_{i}, \quad x_{i}^{\prime}=\rho_{i} y_{i}^{\prime} \quad(i=1, \cdots, n)
$$

with a proper determination of the $\rho_{i}$, restoring for convenience the letter $x$ in place of $y$ after the transformation. This change does not affect our canonical substitution $S_{1}$ nor the values of the $a_{i i}$ in $S$, while the new value of $a_{i j}$ differs from the old value by the factor $\rho_{j} / \rho_{i}$. Also the values of the principal minors of (7) and the characteristic equation (3) remain unaltered. We will so choose $\rho_{1}, \rho_{2}$ that in the substitution $S$ of the preceding paragraph, for which $a_{12} a_{21} \neq 0$, we will have $a_{12}=\bar{a}_{21}$ in case 1 and $a_{12}=-\bar{a}_{21}$ in case 2. It follows then from (13) that we have simultaneously $b_{12}=\bar{b}_{21}$ in case 1 , $b_{12}=-\bar{b}_{21}$ in case 2 for every substitution $S^{\prime}$ of the group.

Next take any odd value of $i$ between 1 and $2 m$ and choose two substitutions $S, S^{\prime}$ for which $b_{1 i} a_{i 1}$ is not zero. Then in consequence of (12) $a_{i+1,2}$ and $b_{2, i+1}$ are not zero. By properly choosing $\rho_{i+1} / \rho_{i}$ we may make $a_{i 1}$ and $a_{i+1,2}$ in $S$ the conjugates of one another. If then $S^{\prime}$ denotes any substitution whatsoever in the group (including $S$ itself), it follows from (12) that for all substitutions $S^{\prime}$ we have simultaneously $b_{1 i}=\bar{b}_{2, i+1}$ for odd $i<2 m$. Taking an $S^{\prime}$ for which $b_{1 i} \neq 0$, we see again from (12) that for every substitution $S$ of the group we have $a_{i 1}=\bar{a}_{i+1,2}$ for odd $i<2 m$.

Consider next the two coefficients in $S^{\prime} S_{1}^{k} S$

$$
\begin{aligned}
& B_{12}^{(k)}=\lambda_{1}^{k} b_{11} a_{12}+\lambda_{2}^{k} b_{12} a_{22}+\cdots+\lambda_{n}^{k} b_{1 n} a_{n 2}, \\
& B_{21}^{(k)}=\lambda_{1}^{k} b_{21} a_{11}+\lambda_{2}^{k} b_{22} a_{21}+\cdots+\lambda_{n}^{k} b_{2 n} a_{n 1} .
\end{aligned}
$$

These are conjugate imaginaries in case 1 and negative conjugates, in case 2. If we set $k=0,1, \cdots, n-1$, we have two systems of equations linear in $b_{1 i} a_{i 2}$ and $b_{2 i} a_{i 1}$ respectively. Reasoning in exactly the same manner as above for equations (11), we obtain the following relations between any two substitutions of our group:

$$
\begin{aligned}
& b_{1 i} a_{i 2}=\bar{b}_{2, i+1} \bar{a}_{i \cdot 1,1} \\
& b_{1 i} a_{i 2}=-\bar{b}_{2, i+1} \bar{a}_{i+1,1} \\
& b_{2 i} a_{i 1}=\bar{b}_{1, i+1} \bar{a}_{i+1,2} \\
& b_{2 i} a_{i 1}=-\bar{b}_{1, i+1} \bar{a}_{i+1,2} \\
& b_{1 i} a_{i 2}=\bar{b}_{2 i} \bar{a}_{i 1} \\
& b_{1 i} a_{i 2}=-\bar{b}_{2 i} \bar{a}_{i 1}
\end{aligned}
$$

(case 1)

(case 2) (odd $i<2 m$ )

(odd $i<2 m$ )

$(i>2 m)$ 
For odd $i<2 m$ our normalization has already made $a_{i 1}=\bar{a}_{i+1,2}$, $\bar{b}_{1 i}=b_{2, i+1}$. If, therefore, we select for (17) a substitution $S$ in which the former pair is not zero and for (16) a substitution $S^{\prime}$ in which the latter pair is not, we get from these equations $b_{2 i}=\bar{b}_{1, i+1}, a_{i 2}=\bar{a}_{i+1,1}$ in case 1 , and $b_{2 i}=-\bar{b}_{1, i+1}, a_{i 2}=-\bar{a}_{i+1,1}$ in case 2 . These results apply to any substitutions $S^{\prime}, S$ in our group. Lastly, if we take a pair of substitutions with $b_{1 i} a_{i 1} \neq 0$ and divide (18) by (14), we get

$$
\frac{a_{i 2}}{a_{i 1}}=\frac{\bar{a}_{i 1}}{\bar{a}_{i 2}} \quad(i>2 m, \text { case } 1),
$$

while in case 2 these two ratios must be the negative of each other. But the latter is impossible, since a quantity cannot equal the negative of the conjugate of its reciprocal. We thus reach the important conclusion that case 2 is possible only when $i=2 m$.

In case 1 equation (19) makes $\left|a_{i 1}\right|=\left|a_{i 2}\right|$. We have not yet disposed of $\rho_{i}$ for $i>2 m$, and we will now so take it that the arguments of $a_{i 1}$ and $a_{i 2}$ in the particular $S$ under consideration shall be the negative of each other. Then we have $\bar{a}_{i 1}=a_{i 2}$, whence it follows by (18) that in case 1 we have $b_{2 i}=\bar{b}_{1 i}(i>2 m)$ for every substitution $S^{\prime}$ in the group. By taking next an $S^{\prime}$ with $b_{1 i} \neq 0$, it follows again from (18) that for every substitution $S$ in the group we have $a_{i 2}=\bar{a}_{i 1}(i>2 m)$ in case 1 .

We have thus completely determined the character of the first two rows and columns of the coefficients (7) of every substitution in our non-loxodromic group. The character of the remaining rows and columns may now be rapidly fixed as follows. We know that for odd $j<2 m$ the coefficients

$$
\begin{aligned}
B_{1 j}^{(k)} & =\lambda_{1}^{k} b_{11} a_{1 j}+\lambda_{2}^{k} b_{12} a_{2 j}+\cdots+\lambda_{n}^{k} b_{1 n} a_{n j}, \\
B_{2}^{(k)}, j+1 & =\lambda_{1}^{k} b_{21} a_{1, j+1}+\lambda_{2}^{k} b_{22} a_{2, j+1}+\cdots+\lambda_{n}^{k} b_{2 n} a_{n, j+1}
\end{aligned}
$$

in $S^{\prime} S_{1}^{k} S$ are conjugate imaginary in both cases 1 and 2 . Putting $k=0,1$, $\cdots, n-1$, we have two systems of equations for the $n$ quantities $b_{1 i} a_{i j}$ and $b_{2 i} a_{i, j+1}(i=1, \cdots, n)$ respectively. Since $\lambda_{i}^{k}, \lambda_{i+1}^{k}$ are conjugate imaginaries for odd $i<2 m$ and $\lambda_{i}$ is real for $i>2 m$, it follows in the same manner as before that we have the following pairs of conjugate imaginaries:

$$
\begin{aligned}
& b_{1 i} a_{i j} \text { and } b_{2, i+1} a_{i+1, j+1} \\
& b_{2 i} a_{i, j+1} \text { and } b_{1, i+1} a_{i+1, j} \\
& b_{1 i} a_{i j} \text { and } b_{2 i} a_{i, j+1}
\end{aligned}
$$$$
\text { (odd } j<2 m \text { and odd } i<2 m \text { ) }
$$$$
\text { (odd } j<2 m \text { and } i>2 m \text { ). }
$$

Now for odd $i<2 m$ we had already $\bar{b}_{1 i}=b_{2, i+1}$ in both cases 1 and 2 , $b_{1, i+1}=\bar{b}_{2 i}$ in case 1 and $b_{1, i+1}=-\bar{b}_{21}$ in case 2 ; while for $i>2 m$ we had $\bar{b}_{1 i}=b_{2 i}$ in case 1 . Since also by our Fundamental Hypothesis we could select $S^{\prime}$ so that a prescribed one of these quantities should not be zero, it follows that for every substitution $S$ of our group we have the relations:

Trans. Am. Math. Soc. 18. 
Case 1.

$$
\begin{aligned}
\bar{a}_{i j} & =a_{i+1, j+1} & & (\text { odd } j<2 m ; \text { odd } i<2 m) \\
\bar{a}_{i, j+1} & =a_{i+1, j} & & (\text { odd } j<2 m ; \text { odd } i>2 m) \\
\bar{a}_{i j} & =a_{i, j+1} & &
\end{aligned}
$$

Case 2.

$$
\begin{aligned}
\bar{a}_{i j} & =a_{i+1, j+1} \\
\bar{a}_{i, j+1} & =-a_{i+1, j+1}
\end{aligned}
$$

The character of the coefficients has just been completely fixed for case 2 and for the first $2 m$ columns of (7) in case 1 . In case 2 the determinant (7) consists of $m^{2}$ blocks of form (6), as stated in the introduction.

It remains only to fix in case 1 the law of the coefficients subsequent to the $2 m$ th column of (7). Now it has already been found that $B_{1 j}^{(k)}$ and $B_{2 j}^{(k)}$ are conjugates for $j>2 m$, and their values will be given by (20) if the subscript $j+1$ in the second equation is replaced throughout by $j$. Taking then $k=0,1, \cdots, n-1$ in the two equations of (20) and arguing in the same manner as before, we obtain for odd $i<2 m$ the conjugate imaginary pairs $b_{1 i} a_{i j}$ and $b_{2, i+1} a_{i+1, j}, b_{2 i} a_{i j}$ and $b_{1, i+1} a_{i+1, j}$, while for $i>2 m$ we have as conjugates $b_{1 i} a_{i j}$ and $b_{2 i} a_{i j}$. For the given odd $i<m$ a substitution $S^{\prime}$ can be employed for which the conjugate elements $b_{1 i}, b_{2, i+1}$ or $b_{2 i}, b_{1, i+1}$ are not zero, and for $i>2 m$ a substitution for which the conjugates $b_{1 i}, b_{2 i}$ are not. Hence, in every substitution $S$ of our group, $a_{i j}$ and $a_{i+1, j}$ are conjugates for odd $i<2 m, j>2 m$, while for $i>2 m, j>2 m$ every $a_{i j}$ is self-conjugate and consequently real.

The completed result which has thus been reached in case 1 may now be summed up as follows. On the assumption that $S_{1}$ possesses distinct characteristic roots $\lambda_{i}$ and at least one pair of roots which are conjugate imaginaries, a normal form for a non-loxodromic group satisfying our Fundamental Hypothesis has been obtained in which every substitution has the following structure. Its determinant (7) consists of four sections. The minor common to the first $2 m$ rows and columns is made up of $m^{2}$ blocks of form

$$
\left|\begin{array}{ll}
a_{i j} & a_{i, j+1} \\
\bar{a}_{i, j+1} & \bar{a}_{i j}
\end{array}\right|
$$

(odd $i$ and $j$ ),

and the minor common to the last $n-2 m$ rows and columns contains only real elements. For $i<2 m, j>2 m$ the elements occur in conjugate pairs $a_{i j}, a_{i+1, j}\left(\right.$ odd $i$ ), and for $i>2 m, j<2 m$ in conjugate pairs $a_{i j}, a_{i, j+1}$ (odd $j$ ). This normal form corresponds to the form $\left(3^{\prime}\right)$ given in the introduction for $m=1$. The simple change of variable

$$
\begin{aligned}
x_{2 i-1} & =y_{2 i-1}+i y_{2 i}, \quad x_{2 i}=y_{2 i-1}-i y_{2 i} \quad & & (i<m), \\
x_{i} & =y_{i} & & (i>2 m)
\end{aligned}
$$


throws the group at once into a second and simpler normal form in which the coefficients are all real.

When $m=0$, the above derivation of the group fails, but the real normal form just indicated is still valid and can be rapidly derived as follows. By hypothesis, all the characteristic roots $\lambda_{i}$ of $S_{1}$ are now real as well as distinct, and accordingly in every substitution (2) of the group all the $a_{i i}$ are real. Since the left members of the system obtained by putting $k=0,1, \cdots, n-1$ in the first equation of (11) are real, all the $b_{1 i} a_{i 1}$ resulting from the solution of the system must be real. Further, by our Fundamental Hypothesis, for each value of $i$ there is some $S^{\prime}$ for which $b_{1 i}$ is not zero, and in this particular $S^{\prime}$ by a change of variable (15) with the proper value of $\rho_{i} / \rho_{1}$ we may make $b_{1 i}(i \neq 1)$ real. It follows then that in every substitution $S$ of the group $a_{i 1}$ is real. By choosing next an $S$ having $a_{i 1} \neq 0$, it follows again from the reality of $b_{1 i} a_{i 1}$ that every substitution $S^{\prime}$ of the group has a real $b_{1 i}$. In this wise all the elements of the first row and column of (7) may be made simultaneously real for all substitutions of the group. Turning next to the system of equations obtained by putting $k=0,1, \cdots, n-1$ in the first equation of (20), we argue in the same manner that every $b_{1 i} a_{i j}$ is real and hence every $a_{i j}$. Thus by the simple change of variable (15) we can throw the group into a normal form in which all of its substitutions are real.

We turn now to consider the significance of our Fundamental Hypothesis that for each value of $i$ there is some substitution of the group for which $a_{1 i} \neq 0$. Make for a moment the contrary hypothesis and suppose that for some fixed $j$ we have $a_{1 j}=0$ in every substitution of the group. ${ }^{*}$ Then in (20) we have $B_{1 j}^{(k)}=0$ for this value of $j$. Taking $k=0,1, \cdots, n-1$, we have a homogeneous system of equations giving $b_{1 i} a_{i j}=0(i=1, \cdots, n)$ for any two substitutions $S, S^{\prime}$ of the group. Hence for any value of $i$ we must have either $b_{1 i}=0$ for every substitution $S^{\prime}$ of the group or $a_{i j}=0$ for every substitution $S$ of the group. Suppose now that there are exactly $r$ values of $j$ for which $a_{1 j}$ is zero in every substitution of the group. Then for each of these $r$ values of $j$ and for any other value of $i$ not one of these $r$ values we must have $a_{i j}=0$ in every substitution of the group. There will be no loss of generality if we assume that in the last $r$ columns of (7) and in no others we have $a_{1 j}=0$, since this can be attained $\dagger$ by merely assigning the subscripts properly to our $n$ variables $x_{i}$. Then all the elements of (7) common to these columns and to the first $n-r$ rows will be zero. Consequently the first $n-r$ equations of (2) contain only the variables $x_{1}, \cdots, x_{n-r}$. This reveals that the space of $r-1$ (complex) dimensions (or a point, if $r=1$ ) obtained

*If some of the $a_{i_{1}}$ are zero for every substitution of the group, a parallel reasoning applies with like result.

†Unless $a_{11}=0$ for every substitution, when we use the first and the last $r-1$ columns in place of the last $r$ columns. 
by putting these coördinates equal to zero is carried over into itself by all substitutions of the group. Conversely, it is evident that if this is the case, the elements of (7) common to the last $r$ columns and the first $n-r$ rows must be zero for all substitutions of the group. The significance of our Fundamental Hypothesis is therefore to forbid that all substitutions of the group should have such an invariant space when $S_{1}$ is taken in form (8).

The hyperplanes $x_{i}=0$ are the only invariant hyperplanes of $S_{1}$ since the multipliers $\lambda_{i}$ in (8) are by hypothesis all distinct. The linear spaces common to $n-r$ of these invariant hyperplanes are also invariant under $S_{1}$, and it is readily seen that they are the only spaces of $r-1$ dimensions invariant under $S_{1}$ which are common to $n-r$ hyperplanes. For consider an invariant space which is defined by linearly independent hyperplanes

$$
u_{i} \equiv \sum_{j=1}^{n} c_{i j} x_{j}=0 \quad(i=1, \cdots, n-r) .
$$

If we take these planes as our coördinate planes along with $r$ other independent hyperplanes, then $S_{1}$ in terms of the new $u$-coördinates will be specified by $n$ equations of form (2) with the $u_{i}$ in place of the $x_{i}$. In the corresponding determinant (7) the elements common to the last $r$ columns and the first $n-r$ rows must be zero since the space common to the hyperplanes $u_{i}=0$ $(i=1, \cdots, n-r)$ is invariant. Accordingly there will be then $n-r$ linearly independent combinations of $u_{1}, \cdots, u_{n-r}$ corresponding to $n-r$ characteristic roots $\lambda_{i}$, and these, except for constant factors, must be identical with $n-r$ coördinates $x_{i}$ which figure in (8). Hence the invariant space common to the hyperplanes $u_{i}=0(i=1, \cdots, n-r)$ can also be specified by putting $n-r$ coördinates $x_{i}$ equal to zero. We thus see that our Fundamental Hypothesis is identical with the requirement that the substitutions of our group should not admit a common invariant hyperplane nor an invariant space common to two or more hyperplanes. This requirement is, of course, unaltered when $S_{1}$ with the group is transformed out of its normal form. It corresponds to the condition mentioned in the introduction, that a non-loxodromic group of substitutions (1) should not have a common pole.

Up to the present point the development has involved only the reality of the characteristic equations (3) and in no wise the signs of the determinants (7). The conclusions reached therefore apply not only to groups of nonloxodromic substitutions but likewise to non-singular groups of substitutions whose characteristic equations are real. For the latter also, under the limitations specified, there are just two classes of groups, and these can be transformed into the two normal forms which have been given. Further, the totality of substitutions of either form, with or without the restriction of being nonloxodromic, form a group. This is evident in the case of substitutions of the 
first kind since the totality of real, non-singular substitutions (2) form a group, and the same holds true if the requirement is added that the determinants of the substitutions shall be positive or that they shall be of value +1 . In the next section it will be shown that the totality of non-singular substitutions of the second kind also form a group, in this case necessarily non-loxodromic.

\section{ON THE NATURE OF THE TWO ClASSES OF NON-LOXODROMIC GROUPS}

A real projective substitution (2) of determinant $\Delta$ can be reduced to a form having the determinant unity by dividing each coefficient in (2) by $\Delta^{1 / n}$. If $\Delta$ is positive, a real $n$th root may always be chosen so that the substitution remains in real form, but if $\Delta$ is negative, this is only possible when $n$ is odd. A real projective substitution in space of $n-1$ (real or complex) dimensions is therefore necessarily non-loxodromic when the number of dimensions is even, but when it is odd, only if the determinant of the substitution is positive.

In accordance with $\S 1$ the general non-loxodromic group of the first class, expressed in normal form, consists of all real substitutions (2) of determinant 1 . Hence it comprises all non-loxodromic substitutions which leave invariant the real space of the $n$ homogeneous variables which lies within the complex $S_{n-1}$. When $n-1$ is even, all real and non-singular projective substitutions of the $n$ variables are included. If the group by transformation through any substitution is thrown out of normal form, the real space is converted into an invariant "chain" for the transformed group.

For the special case $n=2$ it is known that the poles of all hyperbolic and parabolic substitutions in a non-loxodromic group of the first class lie upon the invariant real axis or circle, while the poles of any elliptic substitution of the group lie symmetrically with respect to the real axis or circle. We can easily see that there is a corresponding generalization for greater values of $n$. Consider, for example, any non-loxodromic group of the first class which contains a substitution $S$ whose characteristic roots are all real and distinct. Such a substitution will be called hyperbolic since it is the natural generalization of the hyperbolic substitution for $n=2 .^{*}$ Let the group be transformed into its normal real form. Then the hyperbolic substitution becomes a real substitution (2) of unit determinant. To each of our $n$ distinct characteristic roots $\lambda_{i}$ belongs a linear form,

$$
Y_{i}=c_{1} x_{1}+c_{2} x_{2}+\cdots+c_{n} x_{n},
$$

which undergoes the substitution $Y_{i}^{\prime}=\lambda_{i} Y_{i}$ in consequence of $S$. The well known equations for determining the coefficients $c_{i}$ of the form, given in (23) below, show that the ratios of these coefficients are all real. If we equate

${ }^{*}$ It will presently appear that no hyperbolic substitution is included in a non-loxodromic group of our second class. 
these $n$ forms to zero, we obtain a configuration of $n$ real and distinct hyperplanes, and the $n$ vertices of this configuration are the poles of the hyperbolic substitution. The poles of the substitution are accordingly all real, a conclusion which applies simultaneously to all hyperbolic substitutions of the group. Hence in a non-loxodromic substitution of the first class the poles of its hyperbolic substitutions all lie in the invariant chain of the group.

Conversely, if we have a set of hyperbolic substitutions whose poles lie in a cummon chain, they generate a non-loxodromic group. For let the group be so transformed that the chain becomes the real coördinate space. The poles of any hyperbolic substitutions in the group are now real and by a real transformation of the group may be made to be the $n$-coördinate vertices. The substitution then has the real form $x_{i}^{\prime}=\lambda_{i} x_{i}(i=1, \cdots, n)$ and hence must have had also a real form before the last transformation. Consequently all hyperbolic substitutions of the group have been made simultaneously real. Now by hypothesis our hyperbolic substitutions are of unit-determinant; they generate therefore a group consisting only of real substitutions of unitdeterminant,- that is, a group of non-loxodromic substitutions.

Take now the other extreme case in which the characteristic roots of some particular substitution of a non-loxodromic group of the first class are distinct pairs of conjugate imaginaries. Such a substitution will be called restrictedly elliptic and is possible only when $n$ is an even number $2 m$. By transformation of the group the particular substitution can be thrown into the form (8) and be used as our substitution $S_{1}$ of the preceding section. Without change of $S_{1}$ the group was there brought into a first normal form and then was converted into the final real form by the transformation (21). Since the characteristic roots of our substitution are by hypothesis all distinct, the invariant points of (8) were the $x$-coördinate vertices. The transformation (21) converts the invariant point for which $x_{2 i-1}$ is the only non-zero coördinate into a point for which the $y$-coördinates are all zero except $y_{2 i-1}=x_{2 i-1}, y_{2 i}=-i x_{2 i-1}$, while the invariant point having $x_{2 i}$ as its only non-zero coördinate is converted into one for which all the $y$-coördinates are zero except $y_{2 i-1}=x_{2 i}$, $y_{2 i}=i x_{2 i}$. These two points are imaginary points which lie symmetrically with respect to the real space of the $y$-coördinates; i.e., the space for which the ratios of the $2 m$ homogeneous coördinates are real.* Thus the $2 m$ invariant

* If we think of a complex space $S_{k}$ as a real space with $2 k$ real orthogonal axes, two points lie symmetrically with respect to the linear space determined by $r$-coördinate axes if the corresponding $r$-coördinates of the two points agree, while the remaining coördinates of one point differ only in sign from those of the other. Accordingly, in the case before us, if we interpret the $n$ complex coördinates $y_{i}$ as nonhomogeneous coördinates for a real space of $2 n$ dimensions, the $n=2 m$ invariant points of our substitution are obviously $m$ pairs of points symmetrically situated with respect to the linear space of $n$ dimensions which consists of the points for which the $y_{i}$ are all real. When, however, as above, the coördinates are thought of as projective, some one of the $y_{i}$ equated to zero-let us say $y_{n}=0$-specifies the region at 
points of the substitution $S_{1}$ in its final real form are $m$ pairs of points symmetrically situated with respect to this real space of $2 m-1$ dimensions embedded in the complex space of the coördinates having double this number of dimensions. This real space is the invariant real space of the normalized real group in which our substitution is contained. The other "restrictedly elliptic" substitutions of the group, whose characteristic roots are distinct pairs of conjugate imaginaries, have become real simultaneously with $S_{1}$. Now any two real forms into which a given substitution can be transformed are convertible the one into the other by a real linear change of coördinate variables. Such a change in our $y$-coördinates converts a pair of points, symmetrically situated with respect to the real space of the coördinate-ratios and having corresponding coördinates the conjugates of one another, into another like pair of coördinates. Since, furthermore, any restrictedlyelliptic substitution of the group could be made our $S_{1}$, it follows that the invariant points of all restrictedly elliptic substitutions of a normalized nonloxodromic group of the first species consist of pairs of points which lie symmetrically with respect to the real invariant space of the group. If the group is transformed through any substitution $T$, the real invariant space is converted into a chain, and the images of the invariant point-pairs of these substitutions are converted into pairs of points which may be said to lie symmetrically with respect to this chain.

Turn now to the groups of the second class which are more novel and therefore more interesting. We will first prove that any substitution whose determinant consists of $m^{2}$ blocks of form (6) has a real characteristic equation. To establish this it is necessary to show that in its determinant (7) the sum of all the principal minors of any given order $r$ is real. It suffices to prove that the principal minors which are not real occur in conjugate pairs. With this in view let us

(I) exchange each odd row and column of ( 7 ) with the following even row and column, and

(II) change first the signs of all the elements in the even rows and then the signs of the elements in the even columns of $\Delta$.

By these two changes every element of (7) is replaced by its conjugate. Since the determinant is unaltered in value by the two changes, it must be real, being identical with its conjugate. Any principal minor of (7) formed

infinity and the nonhomogeneous coördinates will be $n-1$ ratios $y_{1} / y_{n}, \cdots, y_{n-1} / y_{n}$. The difficulty arising from the presence of pairs of points for which $y_{n}$ equals zero may be removed by regarding such a pair as the limit of another pair with the same coördinates except $y_{n}$, which is replaced by a real number $h$ approaching zero. If, then, for $h \neq 0$ the two points are symmetrically situated with respect to the real space for which the $(n-1)$-coördinate ratios are real, the limiting points $(h=0)$ will also be said to lie symmetrically with respect to this space. 
by deleting with each odd row and column the following even row and column is real for the same reason. Any other principal minor in consequence of $I$ is exchanged with another principal minor, but neither is changed in sign by II. Hence these two minors must be the conjugate of one another, and the substitution has accordingly a real characteristic equation, as stated.

It will be seen later that a non-zero determinant $\Delta$ having the structure (6) must be positive. If therefore the substitution is non-singular, its determinant can be made equal to +1 by dividing all its coefficients by a real $\sqrt[n]{\Delta}$. All non-singular substitutions of form (5) are therefore non-loxodromic.

Consider next the product of any two (non-singular or singular) substitutions $S, S^{\prime}$ of the second class with coefficients $a_{i j}$ and $b_{i j}$ respectively, and with the same pairing of equations in each, as indicated in (5). Since the coefficients of both obey the law (6), we find at once from (10) that $B_{i+1, j+1}=\bar{B}_{i j}$, $B_{i+1, j}=-\bar{B}_{i, j+1}$ for odd $i$ and $j$. Consequently the product of the two substitutions has the same form.

Furthermore, the inverse of a non-singular substitution will also be of like form. In fact, since its determinant (7) is real, it is only necessary to see that the determinant of the cofactors $A_{i j}$ of the elements of (7) consists of blocks of the form (6). Now we have just seen that the changes I and II together replace every element of (7) by its conjugate and therefore every $A_{i j}$ by its conjugate. On the other hand, these changes obviously result in an interchange of $A_{i j}$ and $A_{i+1, j+1}$ for odd $i$ and $j$, also of $A_{i+1, j}$ and $A_{i, j+1}$, with a reversal of sign in the latter case. Hence we have $A_{i+1, j+1}=\bar{A}_{i j}$, $A_{i+1, j}=-\bar{A}_{i, j+1}$. Thus the inverse substitution has the same form, and we conclude that any number of non-singular substitutions (5) of our second kind generate a non-loxodromic group.

We now proceed to examine the nature of the substitutions of our second class. Let $\lambda_{i}$ be a characteristic root of any substitution (2) of the second class. To this there corresponds a linear form

$$
Y_{1}=c_{1} x_{1}+c_{2} x_{2}+\cdots+c_{n} x_{n}
$$$$
(n=2 m) \text {, }
$$

which in consequence of the substitution undergoes the transformation $Y_{1}^{\prime}=\lambda_{i} Y_{1}$, and its coefficients satisfy the equations

$$
\begin{aligned}
& c_{1}\left(a_{11}-\lambda_{i}\right)+c_{2} a_{21}+\cdots+c_{n} a_{n 1}=0, \\
& c_{1} a_{12}+c_{2}\left(a_{22}-\lambda_{i}\right)+\cdots+c_{n} a_{n 2}=0 \text {, } \\
& c_{1} a_{1 n}+c_{2} a_{2 n}+\cdots+c_{n}\left(a_{n n}-\lambda_{i}\right)=0 \text {, }
\end{aligned}
$$

the consistency of which is guaranteed by our characteristic equation. Suppose now every quantity which appears in these equations to be replaced by its conjugate. We get a system of equations for $\bar{c}_{1}, \cdots, \bar{c}_{n}$. Exchange next 
each odd equation in (23) with the following even equation, then each odd column with the following even column, and finally change the signs of the $n$ terms of the resulting even equations. Then by virtue of the fact that $a_{i j}, a_{i+1, j+1}$, also $a_{i+1, j}$ and $-a_{i, j+1}$ are conjugates for odd $i$ and $j$, we get precisely the equations which say that

$$
-Z_{1}=\left(\bar{c}_{2} x_{1}-\bar{c}_{1} x_{2}\right)+\left(\bar{c}_{4} x_{3}-\bar{c}_{3} x_{4}\right)+\cdots+\left(\bar{c}_{n} x_{n-1}-\bar{c}_{n-1} x_{n}\right)
$$

undergoes the transformation $Z_{1}^{\prime}=\bar{\lambda}_{i} Z_{1}$ in consequence of our substitution (2). Thus the pair of linear forms which correspond to a simple pair of conjugate imaginary roots of the characteristic equation have the form (22) and (24). Moreover, the two forms cannot be linearly dependent even when $\lambda_{i}$ is self-conjugate and real, for then we would have

$$
\frac{\bar{c}_{2 i}}{c_{2 i-1}}=-\frac{\bar{c}_{2 i-1}}{c_{2 i}}, \quad \text { or } \quad c_{2 i} \bar{c}_{2 i}+c_{2 i-1} \bar{c}_{2 i-1}=0 \quad(i=1, \cdots, m),
$$

which would make every $c_{i}=0$ in (22). It follows that every real root of the characteristic equation must be at least a double root.

In case the elementary divisors of the characteristic equation are not all simple, there correspond $s$ linear forms to each elementary divisor $\left(\lambda-\lambda_{i}\right)^{s}$ of the $x_{i}$ which in consequence of the substitution undergo the transformation

$$
\begin{aligned}
& Y_{1}^{\prime}=\lambda_{i} Y_{1}, \\
& Y_{2}^{\prime}=Y_{1}+\lambda_{i} Y_{2}, \\
& Y_{3}^{\prime}=Y_{2}+\lambda_{i} Y_{3}, \\
& \cdot \cdot \cdot \cdot \cdot \cdot \cdot \cdot \cdot \\
& Y_{s}^{\prime}=Y_{s-1}+\lambda_{i} Y_{s} .
\end{aligned}
$$

Let us suppose that $Y_{1}$ is given by the right member of (22), and correspondingly $Z_{1}$ by (24). Put

$$
Y_{2}=d_{1} x_{1}+d_{2} x_{2}+\cdots+d_{n} x_{n} \quad(n=2 m) .
$$

By use of (1) and (26) in the second equation of (25) we get

$$
\begin{aligned}
& d_{1}\left(a_{11}-\lambda_{i}\right)+d_{2} a_{21}+\cdots+d_{n} a_{n 1}=c_{1}, \\
& d_{1} a_{12}+d_{2}\left(a_{22}-\lambda_{i}\right)+\cdots+d_{n} a_{n 2}=c_{2} \text {, } \\
& d_{1} a_{1 n}+d_{2} a_{2 n}+\cdots+d_{n}\left(a_{n n}-\lambda_{i}\right)=c_{n} \text {. }
\end{aligned}
$$

Replace every quantity which appears in these equations by its conjugate, exchange each odd equation with the following even equation, then exchange each odd column with the following even column, and finally change the signs of the terms on both sides of the even equations. We get a set of equations which state that the linear form

$$
-Z_{2}^{\prime}=\left(\bar{d}_{2} x_{1}-\bar{d}_{1} x_{2}\right)+\cdots+\left(\bar{d}_{n} x_{n-1}-\bar{d}_{n-1} x_{n}\right)
$$


undergoes the transformation

$$
Z_{2}^{\prime}=Z_{1}+\bar{\lambda}_{i} Z_{2}
$$

in consequence of our substitution. Proceeding next in the same way with the third equation of (25) and so on in turn with each of its succeeding equations, we find that to the $s$ linear forms $Y_{1}, \cdots, Y_{s}$ there correspond also $s$ other linear forms $Z_{1}, \cdots, Z_{s}$, which are related to them in exactly the same way as (24) to (22) and which undergo the transformation

$$
\begin{aligned}
& Z_{1}^{\prime}=\bar{\lambda}_{i} Z_{1}, \\
& Z_{2}^{\prime}=Z_{1}+\bar{\lambda}_{i} Z_{2}, \\
& \cdot \cdot \cdot \cdot \cdot \\
& Z_{s}^{\prime}=Z_{s-1}+\bar{\lambda}_{i} Z_{s},
\end{aligned}
$$

in consequence of the substitution. The coexistence of (29) and (25) indicates that for each elementary divisor $\left(\lambda-\lambda_{i}\right)^{8}$ there is another divisor $\left(\lambda-\bar{\lambda}_{i}\right)^{8}$, and hence gives the following result:

Theorem. The elementary divisors of the characteristic equation of any non-singular substitution (5) occur only in conjugate pairs $\left(\lambda-\lambda_{i}\right)^{s},\left(\lambda-\bar{\lambda}_{i}\right)^{s}$ of equal degree, this conclusion holding also for the real characteristic roots $\lambda_{i}$ to which belong pairs of equal elementary divisors.

Since the determinant of a substitution is the product of the characteristic roots, taken each with its proper multiplicity, the theorem just stated gives at once the following important corollary:

CoRollary. If a non-singular substitution has the form (5), its determinant is positive.

Determinants with real elements satisfying the condition (6) have been previously encountered in some work of Voigt and have been called $D e$ terminants of Voigt by Kowalewski. The proofs* given to show that Voigt's determinant is positive or zero fail altogether to cover the more general determinants here considered in which the elements are complex. As the proof furnished above was indirect, I shall also give a simple and direct proof.

The non-negative character of the determinant is obvious when $m=1$ since it is then $a_{11} \bar{a}_{11}+a_{12} \bar{a}_{12}$. The extension to any other value of $m$ is obtained in the following manner by mathematical induction. Suppose it to have been established for any value of $m$ that all determinants of order $2 m-2$ having the structure (6) are non-negative. Take then any de-

* Kowalewski, Einführung in die Determinantentheorie, $\$ 69$, p. 156. Baltzer's and Drude's proofs are both found in the Göt tinger N a c h ri c h t e n, 1887.

Drude and Kowalewski prove, when the elements of (7) are real, that $\Delta$ is the sum of the squares of two expressions. When the elements are complex, the natural extension would be to replace each square by the product of the expression and its conjugate. But the resulting sum no longer represents $\Delta$. 
terminant (7) of order $2 m$, which obeys the same law, and multiply it by any minor $\Delta_{2 m-2}$ of order $2 m-2$ obtained by deleting from $\Delta$ any odd row and any odd column and the following even row and column. This minor has the structure (6), and there will be no loss of generality in supposing that it consist of the first $2 m-2$ rows and columns of $\Delta$ since the deleted rows and columns may be moved to the bottom and to the right-hand border of $\Delta$ without violating the law of its formation. By a well known identity* we then have

$$
\Delta_{2 m-2} \Delta=A_{2 m-1,2 m-1} A_{2 m, 2 m}-A_{2 m-1,2 m} A_{2 m, 2 m-1} \text {. }
$$

Now we have already observed that $A_{2 m-1,2 m-1}$ and $A_{2 m, 2 m}$ are conjugates, while $A_{2 m-1,2 m}$ and $A_{2 m, 2 m-1}$ are negative conjugates. Hence the right member of (30) is non-negative, being positive unless all four $A_{i j}$ on the right of (30) are zero. If, therefore, a single one of the minors $\Delta_{2 m-2}$ formed in the manner indicated is positive, it follows that $\Delta$ is positive or zero. On the other hand, if every minor $\Delta_{2 m-2}$ is zero, every $A_{i j}$ by (30) must be zero and hence also $\Delta$ itself. Consequently, any determinant consisting of blocks of form (6) must be non-negative. Hence the determinant of any non-singular substitution of form (5) is positive.

Any non-singular substitution in $n$ variables which consists only of pairs of components of form (25) and (29) is a substitution of our second class since it can be thrown into form (5) by alternating the equations of (29) with those of (25). Thus, for example, the alternation of their first two equations gives a matrix of coefficients

having the structure (6).

$$
\left\|\begin{array}{llll}
\lambda & 0 & 0 & 0 \\
0 & \frac{\lambda}{\lambda} & 0 & 0 \\
1 & 0 & \lambda & 0 \\
0 & 1 & 0 & \bar{\lambda}
\end{array}\right\|
$$

In establishing the existence of the linear forms $Y_{i}, Z_{i}$ for any non-singular substitution of the second class, we implicitly reduced it to canonical form. If we regard our $m$ pairs of equations of form (22) and (24) as a change of variable, the substitution in terms of the new variables consists of pairs of components (25) and (29). Now the transforming equations (22) and (24) are of exactly the same form as (5). Hence any substitution of the second class can be thrown into canonical form by transformation through an appropriately chosen transformation of the same class. Since all the transformations (5) form a group, this can be also done without destroying the form (5) of a non-loxodromic group in which it is contained.

An important property of substitutions (5) of our second class is to convert a pair of points

$$
P=\left(x_{1}, x_{2} ; \cdots ; x_{2 m-1}, x_{2 m}\right), \quad Q=\left(-\bar{x}_{2}, \bar{x}_{1} ; \cdots ;-\bar{x}_{2 m}, \bar{x}_{2 m-1}\right)
$$

\footnotetext{
* Muir's Theory of Determinants, $\S 90$.
} 
into another similar pair. By direct substitution in (5) it will be found at once that when the substitution converts $P$ into $P^{\prime}=\left(x_{2 i-1}^{\prime}, x_{2 i}^{\prime}\right), i=1, \cdots, m$, it also converts $Q$ into $Q^{\prime}=\left(-\bar{x}_{2 i}^{\prime}, \bar{x}_{2 i-1}^{\prime}\right)$. A pair of such points we will speak of as conjugate with respect to the substitution or group.

We will now prove, conversely, that if any substitution (2) in our $2 m$ variables has the property of carrying every pair of conjugate points into a pair of conjugate points, it must be one of our second class having the form (5). Consider the first two equations of (2) which, for convenience, we will write

$$
\begin{aligned}
& x_{1}^{\prime}=a_{1} x_{1}+a_{2} x_{2}+\cdots+a_{n} x_{n}, \\
& x_{2}^{\prime}=b_{1} x_{1}+b_{2} x_{2}+\cdots+b_{n} x_{n} \quad(n=2 m) .
\end{aligned}
$$

The coefficients of either equation will, in general, be determined by the requirement that $m$ pairs of conjugate points $P_{i}, Q_{i}$ shall be transformed into conjugate pairs $P_{i}^{\prime}, Q_{i}^{\prime}$. We get thus the following sets of equations for the determination of our coefficients $a_{i}, b_{i}$ :

$$
\begin{aligned}
x_{i 1}^{\prime}=a_{1} x_{i 1}+a_{2} x_{i 2}+\cdots+a_{2 m-1} x_{i, 2 m-1}+a_{2 m} x_{i, 2 m}, \\
-\bar{x}_{i 2}^{\prime}=-a_{1} \bar{x}_{i 2}+a_{2} \bar{x}_{i 1}+\cdots-a_{2 m-1} \bar{x}_{i, 2 m}+a_{2 m} \bar{x}_{i, 2 m-1} \\
\quad(i=1, \cdots, m), \\
x_{i 2}^{\prime}=b_{1} x_{i 1}+b_{2} x_{i 2}+\cdots+b_{2 m-1} x_{i, 2 m-1}+b_{2 m} x_{i, 2 m}, \\
\bar{x}_{i 1}^{\prime}=-b_{1} \bar{x}_{i 2}+b_{2} \bar{x}_{i 1}+\cdots-b_{2 m-1} \bar{x}_{i, 2 m}+b_{2 m} \bar{x}_{i, 2 m-1} \\
(i=1, \cdots, m) .
\end{aligned}
$$

The determinants of these two sets of equations are identical and must be positive or zero since they are composed of blocks of form (6). To exclude the value zero, it will be assumed that $m$ pairs of conjugate points $P, Q$ are taken which do not lie in a common hyperplane of our complex $S_{n-1}$. The numerators of the values obtained for $a_{1}$ and $b_{2}$ are determinants differing only in their first two columns which consist of $m$ blocks

$$
\left\|\begin{array}{rr}
x_{i 1}^{\prime} & x_{i 2} \\
-\bar{x}_{i 2}^{\prime} & \bar{x}_{i 1}
\end{array}\right\|, \quad \mid \begin{array}{rr}
x_{i 1} & x_{i 2}^{\prime} \\
-\bar{x}_{i 2} & \bar{x}_{i 1}^{\prime}
\end{array} \|,
$$

respectively, while the corresponding blocks in the numerators of $a_{2}$ and $b_{1}$ are

$$
\left\|\begin{array}{rr}
x_{i 1} & x_{i 1}^{\prime} \\
-\bar{x}_{i 2} & -\bar{x}_{i 2}^{\prime}
\end{array}\right\|, \quad\left\|\begin{array}{ll}
x_{i 2}^{\prime} & x_{i 2} \\
\bar{x}_{i 1}^{\prime} & \bar{x}_{i 1}
\end{array}\right\| .
$$

The remaining pairs of columns are identical for all four numerators and are of structure (6). If now in the numerator of $b_{2}$ we exchange each odd row and column with the following even row and column and change first the signs of the even columns and then of the even rows, each element of the numerator determinant is converted into the conjugate of the corresponding 
element in the numerator of $a_{1}$. Consequently $b_{2}=\bar{a}_{1}$. A like manipulation of the numerator of $b_{1}$ gives $b_{1}=-\bar{a}_{2}$. In the same way we obtain $b_{2 i}=\bar{a}_{2 i+1}$, $b_{2 i+1}=-\bar{a}_{2 i}$. Hence the first two equations of the substitution must have the structure (6). The reasoning applies to all its succeeding pairs of equations. Thus the property of converting pairs of conjugate points into conjugate pairs completely characterizes the second class of substitutions.

One immediate corollary of this result is that the invariant points of a substitution of the second class consist of pairs of conjugate points, for clearly if one point of a conjugate pair is invariant, the other must be. The geometrical interpretation of this is well known for $m=1$. If, namely, complex coördinates are given to the points of a sphere in the proper way, the formula (4) represents a rotation of the sphere about the diameter passing through the two invariant points of the sphere, and the coördinates* of any two diametrically opposite points are $z,-1 / \bar{z}$, or, in homogeneous notation, $\left(z_{1}, z_{2}\right)$ and $\left(-\bar{z}_{2}, \bar{z}_{1}\right)$.

It has thus appeared that our second class of groups generalizes the characteristic form and property of the familiar elliptic rotation groups for $m=1$. For $m>1$ it accordingly seems fitting to apply the term elliptic to any substitutions of our second kind, that is, to the substitutions whose elementary divisors occur only in conjugate imaginary or duplicate real pairs. The general elliptic group will then consist of all non-singular substitutions of the second class with the same pairing of the coördinates, as indicated in equation (5). A subclass will comprise the restrictedly elliptic substitutions whose characteristic roots are $n$ distinct pairs of conjugate imaginaries.

* Cf. Klein's Vorlesungen über das Ikosaeder, pp. 32-34.

UNIVERSITY OF WISCONSIN, Madison, Wis. 